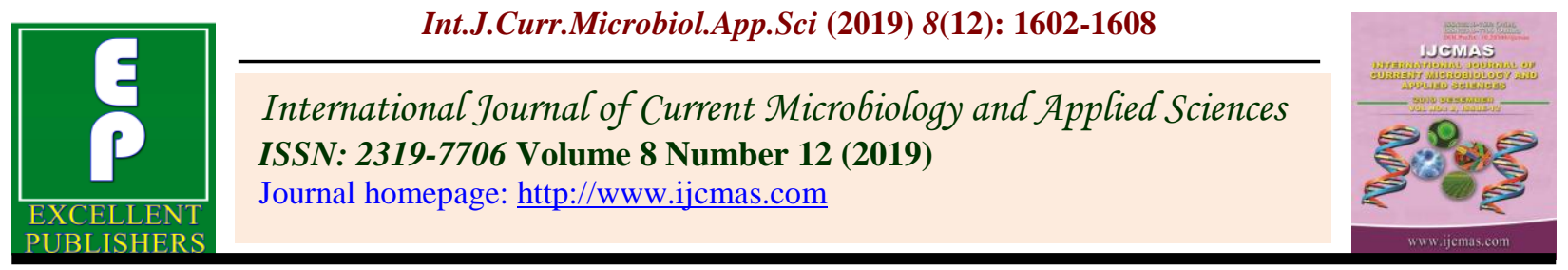

Original Research Article

https://doi.org/10.20546/ijcmas.2019.812.192

\title{
Estimation of Correlation and Path Analysis for Quantitative traits in Chickpea (Cicer arientinum $\mathrm{L}$.)
}

\author{
Bijjamwar Makarand*, G. M. Lal and I. Karthik Manikanta Sai \\ Department of Genetics and Plant Breeding, Naini Agricultural Institute, Sam Higginbottom \\ University of Agriculture, Technology and Sciences, Prayagraj, Uttar Pradesh, India \\ *Corresponding author
}

\section{A B S T R A C T}

Keywords

Chickpea (Cicer

arietinum $\mathrm{L}$.),

Correlation and

Path analysis

Article Info

Accepted:

12 November 2019

Available Online:

10 December 2019
A study was carried out in the fields of Department of Genetics and Plant Breeding, Sam Higginbottom University of Agriculture, Technology and Sciences, Prayagraj, Uttar Pradesh during 2018-2019. Fifty one genotypes of chickpea were evaluated for means and components of variability, heritability, genetic advance and interrelationships for various parameters, direct and indirect effect of various parameters for seed yield. Characters like days to maturity, followed by number of pods per plant, number of primary branches, number of secondary branches, number of pods per plant, number of seeds per pod, days to $50 \%$ flowering etc.. Grain yield had high positive and significant association with days to 50\% flowering, plant height, number of primary branches, number of secondary branches, number of pods per plant, number of seeds per plant, biological yield and harvest index. Path coefficient analysis showed maximum direct contribution towards grain yield per plant with biological yield followed by harvest index.

\section{Introduction}

Chickpea (Cicer arietinum L.) is an integral part of an Indian agriculture since time immemorial, because of only its intrinsic value in terms of higher protein content, carbohydrates, minerals, nitrogen fixing ability and indispensability as alternative crop for crop diversification. Chickpea (Cicer arietinum $L$.) occupies a prime position among the pulses in the country with a maximum hectarage, production and its high nutritive value. It is known to have originated in western Asia. It belongs to genus Cicer and tribe Viciacae, sub-order Papilionaceae of order Leguminoseae (Bentham and Hooker, 1870). Total out of 39 known species are distributed mainly in central and western asia, two are found to be cultivated in india, viz; Cicer arietinum $(2 \mathrm{n}=14)$ which is most widely cultivated and Cicer soongaricum ( $2 \mathrm{n}$ $=16$ ) cultivated in western temperate and alpine regions (9000-15000 ft.in altitude) of the Himalaya. Southwest Asia and the Mediterranean particularly South -East Turkey and Syria are considered as the primary centre of origin of chickpea Ethiopia is regarded as secondary centre of origin. Chickpea contains 
about 17.7 to 38.5 per cent protein and 56.5 per cent carbohydrates besides ash, calcium, phosphorus and iron. Exudation from leaves called "amb" contains oxalic and malic acids which possess medicinal value and used for intestinal disorders, stomach aches, blood purification etc., germinating seeds are prophylactic against scurvy disease. The whole grains are also eaten as raw, boiled or roasted.

Chickpea is one of the most important Rabi pulse crops in Asia. India is largest producer (25\%), importer $(20 \%)$ and consumer (20\%) of pulses in the world. In pulses, chickpea accommodates third position in the world.

\section{Materials and Methods}

Fifty one genotypes of chickpea including check were evaluated during Rabi 2018-2019 following $\mathrm{RBD}$ with three replications. The research was conducted at the Field Experimentation Centre of Department of Genetics and Plant Breeding, Prayagraj, situated in the "Middle Gangetic Plains" which falls under agro-climatic zone. All the recommended cultural practices were adopted to maintain a healthy crop growth. Data regarding yield and twelve attributing traitsviz, days to $50 \%$ flowering, plant height (cm), days to maturity, number of primary branches, number of secondary branches, number of seeds per pod, number of pods per plant, number of seeds per plant, seed index (g), days to $50 \%$ pod setting, biological yield (g), harvest index (\%) and grain yield per plant were recorded for all the chickpea genotypes.

The character association estimated from variance and co variance components as given. While the direct and indirect effects of component traits on grain yield were measured by path analysis.

\section{Results and Discussion}

Correlation analysis (Table 1) revealed that the grain yield per plant showed positive correlation to Days to $50 \%$ flowering showed positive significant association with grain yield per plant $(0.517 * *)$, Days to maturity showed positive significant association with number of secondary branches $\left(0.167^{*}\right)$, days to $50 \%$ pod setting $(0.381 * *)$, Plant height showed positive significant association with number of primary branches $\left(0.164^{*}\right)$, number of secondary branches $\left(0.518^{* *}\right)$, seed index $\left(0.370^{* *}\right)$, biological yield $(0.390 * *)$ and grain yield per plant $(0.349 * *)$, Number of primary branches showed positive significant association with number of secondary branches $\left(0.484^{* *}\right)$, number of pods per plant $(0.426 * *)$, number of seeds per plant $\left(0.255^{* *}\right)$, days to $50 \%$ pod setting $(0.201 * *)$, biological yield $(0.432 * *)$, harvest index $\left(0.236^{* *}\right)$ and grain yield per plant $\left(0.349^{* *}\right)$.

Number of secondary branches showed positive significant association with number of pods per plant $\left(0.365^{* *}\right)$, number of seeds per plant $(0.351 * *)$, biological yield $(0.640 * *)$, harvest index $(0.347 * *)$ and grain yield per plant $\left(0.643^{* *}\right)$, Number of pods per plant showed positive significant association with number of seeds per plant $(0.738 * *)$, biological yield $\left(0.638^{* *}\right)$, harvest index $\left(0.245^{* *}\right)$ and grain yield per plant $(0.637 * *)$, Number of seeds per plant showed positive significant association with number of seeds per pod $\left(0.255^{* *}\right)$, biological yield $\left(0.570^{* *}\right)$, harvest index $\left(0.178^{* *}\right)$ and grain yield per plant $(0.560 * *)$, Number of seeds per pod showed positive significant association with harvest index $\left(0.231^{* *}\right)$, Biological yield showed positive significant association with harvest index $\left(0.493^{* *}\right)$ and grain yield per hill $(0.997 * *)$. Harvest index showed positive significant association with grain yield per plant $(0.559 * *)$. 
Table.1 Estimation of genotypic and phenotypic correlation coefficient of 13 yield component traits with seed yield in chickpea during rabi, 2018

\begin{tabular}{|c|c|c|c|c|c|c|c|c|c|c|c|c|c|c|c|}
\hline $\begin{array}{l}\text { Sr. } \\
\text { no }\end{array}$ & Character & & $\begin{array}{c}\text { Days to } \\
\mathbf{5 0 \%} \\
\text { Flowering }\end{array}$ & $\begin{array}{l}\text { Days to } \\
\text { Maturity }\end{array}$ & $\begin{array}{c}\text { Plant } \\
\text { height }\end{array}$ & $\begin{array}{l}\text { Primary } \\
\text { branches }\end{array}$ & $\begin{array}{c}\text { Secondary } \\
\text { branches }\end{array}$ & $\begin{array}{l}\text { No. Pods } \\
\text { per plant }\end{array}$ & $\begin{array}{l}\text { No. seed per } \\
\text { plant }\end{array}$ & $\begin{array}{l}\text { No. seeds } \\
\text { per pod }\end{array}$ & $\begin{array}{l}\text { Seed } \\
\text { Index }\end{array}$ & $\begin{array}{l}\text { Days to } \\
50 \% \text { Pod } \\
\text { Setting }\end{array}$ & $\begin{array}{c}\text { Biologica } \\
1 \text { yield }\end{array}$ & $\begin{array}{l}\text { Harvest } \\
\text { Index }\end{array}$ & $\begin{array}{c}\text { Seed } \\
\text { yield/plant }\end{array}$ \\
\hline \multirow[t]{2}{*}{1} & \multirow{2}{*}{$\begin{array}{c}\text { Days to } 50 \% \\
\text { Flowering }\end{array}$} & $\mathrm{P}$ & 1 & 0.038 & -0.119 & $-0.169^{*}$ & $-0.307^{* *}$ & $-0.410^{* *}$ & $-0.192^{*}$ & 0.129 & -0.087 & $0.310^{* *}$ & $-0.495^{* *}$ & -0.11 & $0.417 * *$ \\
\hline & & $\mathrm{G}$ & 1 & 0.113 & -0.155 & $-0.204^{*}$ & $-0.358^{* *}$ & $-0.479^{* *}$ & $-0.221^{* *}$ & 0.15 & -0.12 & 0.119 & $-0.586^{* *}$ & $-0.242^{* *}$ & $0.517 * *$ \\
\hline \multirow[t]{2}{*}{2} & \multirow{2}{*}{$\begin{array}{l}\text { Days to } \\
\text { Maturity }\end{array}$} & $\mathrm{P}$ & & 1 & 0.084 & 0.047 & 0.155 & $-0.162^{*}$ & -0.128 & $-0.306^{* *}$ & 0.062 & $0.200^{*}$ & 0.084 & 0.063 & 0.091 \\
\hline & & G & & 1 & 0.12 & 0.055 & $0.167^{*}$ & $-0.172^{*}$ & -0.132 & $-0.365^{* *}$ & 0.074 & $0.381^{* * *}$ & 0.092 & 0.114 & 0.098 \\
\hline \multirow[t]{2}{*}{3} & \multirow{2}{*}{$\begin{array}{l}\text { Plant } \\
\text { height }\end{array}$} & $\mathrm{P}$ & & & 1 & 0.102 & $0.346^{* *}$ & -0.096 & -0.085 & -0.057 & $0.226^{* *}$ & -0.066 & $0.209^{* *}$ & 0.003 & $0.197^{*}$ \\
\hline & & $\mathrm{G}$ & & & 1 & $0.164^{*}$ & $0.518^{* *}$ & $-0.167^{*}$ & -0.113 & -0.077 & $0.370^{* *}$ & 0.045 & $0.390^{* *}$ & $-0.169^{*}$ & $0.349^{* *}$ \\
\hline \multirow[t]{2}{*}{4} & \multirow{2}{*}{$\begin{array}{l}\text { Primary } \\
\text { branches }\end{array}$} & $\mathrm{P}$ & & & & 1 & $0.458^{* *}$ & $0.403^{* *}$ & $0.238^{* *}$ & -0.07 & -0.079 & 0.113 & $0.401^{* *}$ & 0.117 & $0.403^{* *}$ \\
\hline & & $\mathrm{G}$ & & & & 1 & $0.484^{* *}$ & $0.426^{* *}$ & $0.255^{* *}$ & -0.091 & -0.079 & $0.201^{*}$ & $0.432^{* *}$ & $0.236^{* *}$ & $0.437^{* *}$ \\
\hline \multirow[t]{2}{*}{5} & \multirow{2}{*}{$\begin{array}{c}\text { Secondary } \\
\text { branches }\end{array}$} & $\mathrm{P}$ & & & & & 1 & $0.362^{* *}$ & $0.347^{* *}$ & -0.075 & 0.011 & -0.038 & $0.617^{* *}$ & $0.223^{* *}$ & $0.622^{* *}$ \\
\hline & & $\mathrm{G}$ & & & & & 1 & $0.365^{* *}$ & $0.351^{* *}$ & -0.087 & 0.015 & -0.034 & $0.640^{* *}$ & $0.347^{* *}$ & $0.643^{* *}$ \\
\hline \multirow[t]{2}{*}{6} & \multirow{2}{*}{$\begin{array}{l}\text { No. Pods } \\
\text { per plant }\end{array}$} & $\mathrm{P}$ & & & & & & 1 & $0.732^{* *}$ & -0.053 & $-0.239^{* *}$ & -0.119 & $0.619^{* *}$ & $0.175^{*}$ & $0.623^{* *}$ \\
\hline & & $\mathrm{G}$ & & & & & & 1 & $0.738^{* *}$ & -0.061 & $-0.245^{* *}$ & $-0.176^{*}$ & $0.638^{* *}$ & $0.245^{* *}$ & $0.637^{* *}$ \\
\hline \multirow[t]{2}{*}{7} & \multirow{2}{*}{$\begin{array}{l}\text { No. seed } \\
\text { per plant }\end{array}$} & $\mathrm{P}$ & & & & & & & 1 & $0.237^{* *}$ & $-0.435^{* *}$ & -0.124 & $0.554^{* *}$ & 0.13 & $0.549^{* *}$ \\
\hline & & $\mathrm{G}$ & & & & & & & 1 & $0.255^{* *}$ & $-0.451^{* *}$ & $-0.188^{*}$ & $0.570^{* *}$ & $0.178^{*}$ & $0.560^{* *}$ \\
\hline \multirow[t]{2}{*}{8} & \multirow{2}{*}{$\begin{array}{l}\text { No.seeds } \\
\text { per pod }\end{array}$} & $\mathrm{P}$ & & & & & & & & 1 & -0.148 & $-0.195^{*}$ & -0.086 & 0.117 & 0.087 \\
\hline & & $\mathrm{G}$ & & & & & & & & 1 & -0.146 & $-0.345^{* *}$ & -0.101 & $0.231^{* *}$ & 0.103 \\
\hline \multirow[t]{2}{*}{9} & \multirow{2}{*}{$\begin{array}{l}\text { Seed } \\
\text { Index }\end{array}$} & $\mathrm{P}$ & & & & & & & & & 1 & -0.079 & 0.082 & -0.069 & 0.071 \\
\hline & & $\mathrm{G}$ & & & & & & & & & 1 & -0.135 & 0.082 & -0.09 & 0.072 \\
\hline \multirow{2}{*}{$\begin{array}{l}1 \\
0\end{array}$} & \multirow{2}{*}{$\begin{array}{l}\text { Days to } \\
\text { 50\% Pod } \\
\text { Setting }\end{array}$} & $\mathrm{P}$ & & & & & & & & & & 1 & 0.023 & 0.114 & 0.033 \\
\hline & & $\mathrm{G}$ & & & & & & & & & & 1 & 0.06 & 0.099 & 0.057 \\
\hline \multirow{2}{*}{$\begin{array}{l}1 \\
1\end{array}$} & \multirow{2}{*}{$\begin{array}{l}\text { Biological } \\
\text { yield }\end{array}$} & $\mathrm{P}$ & & & & & & & & & & & 1 & $0.256^{* *}$ & $0.989^{* *}$ \\
\hline & & $\mathrm{G}$ & & & & & & & & & & & 1 & $0.493^{* *}$ & $0.997^{* *}$ \\
\hline \multirow{2}{*}{$\begin{array}{l}1 \\
2\end{array}$} & \multirow{2}{*}{$\begin{array}{l}\text { Harvest } \\
\text { Index }\end{array}$} & $\mathrm{P}$ & & & & & & & & & & & & 1 & $0.392^{* *}$ \\
\hline & & $\mathrm{G}$ & & & & & & & & & & & & 1 & $0.559^{* *}$ \\
\hline
\end{tabular}


Table.2 Estimation of path coefficient at genotypic level for 13 yield component traits with grain yield per plant in chickpea during rabi, 2018

\begin{tabular}{|c|c|c|c|c|c|c|c|c|c|c|c|c|c|c|}
\hline No & Character & $\begin{array}{c}\text { Days to } \\
\mathbf{5 0 \%} \\
\text { Flowering }\end{array}$ & $\begin{array}{c}\text { Days to } \\
\text { Maturity }\end{array}$ & $\begin{array}{c}\text { Plant } \\
\text { height }\end{array}$ & $\begin{array}{l}\text { Primary } \\
\text { branches }\end{array}$ & $\begin{array}{l}\text { Secondary } \\
\text { branches }\end{array}$ & $\begin{array}{c}\text { No.Pods } \\
\text { per } \\
\text { plant }\end{array}$ & $\begin{array}{l}\text { No. seed } \\
\text { per } \\
\text { plant }\end{array}$ & $\begin{array}{c}\text { No. } \\
\text { seeds per } \\
\text { pod }\end{array}$ & $\begin{array}{l}\text { Seed } \\
\text { Index }\end{array}$ & $\begin{array}{c}\text { Days to } \\
\mathbf{5 0 \%} \\
\text { Pod } \\
\text { Setting }\end{array}$ & $\begin{array}{c}\text { Biological } \\
\text { yield }\end{array}$ & $\begin{array}{c}\text { Harvest } \\
\text { Index }\end{array}$ & $\begin{array}{l}\text { Seed } \\
\text { yield/ } \\
\text { plant }\end{array}$ \\
\hline 1. & $\begin{array}{c}\text { Days to } \\
50 \% \\
\text { Flowering }\end{array}$ & 0.0134 & 0.0004 & -0.0008 & -0.0020 & 0.0018 & -0.0014 & -0.0036 & -0.0028 & -0.0008 & -0.0016 & 0.5396 & $\begin{array}{c}- \\
0.0253\end{array}$ & 0.51693 \\
\hline 2. & $\begin{array}{l}\text { Days to } \\
\text { Maturity }\end{array}$ & -0.0015 & 0.0036 & 0.0006 & 0.0005 & -0.0008 & -0.0005 & -0.0022 & 0.0067 & 0.0005 & -0.0050 & 0.0846 & 0.0119 & 0.09847 \\
\hline 3. & $\begin{array}{c}\text { Plant } \\
\text { height } \\
\text { (cm) }\end{array}$ & 0.0021 & 0.0004 & 0.0051 & 0.0016 & -0.0026 & -0.0005 & -0.0019 & 0.0014 & 0.0026 & -0.0006 & 0.3590 & $\begin{array}{c}- \\
0.0176\end{array}$ & 0.349 \\
\hline 4. & $\begin{array}{l}\text { Primary } \\
\text { branches }\end{array}$ & 0.0027 & 0.0002 & 0.0008 & 0.0098 & -0.0024 & 0.0012 & 0.0042 & 0.0017 & -0.0005 & -0.0026 & 0.3978 & 0.0246 & 0.43747 \\
\hline 5. & $\begin{array}{c}\text { Secondary } \\
\text { branches }\end{array}$ & 0.0048 & 0.0006 & 0.0027 & 0.0048 & -0.0050 & 0.0011 & 0.0058 & 0.0016 & 0.0001 & 0.0004 & 0.5898 & 0.0362 & 0.64271 \\
\hline 6. & $\begin{array}{l}\text { No. Pods } \\
\text { per plant }\end{array}$ & 0.0064 & -0.0006 & -0.0009 & 0.0042 & -0.0018 & 0.0029 & 0.0121 & 0.0011 & -0.0017 & 0.0023 & 0.5873 & 0.0256 & 0.63697 \\
\hline 7. & $\begin{array}{l}\text { No. seed } \\
\text { per plant }\end{array}$ & 0.0030 & -0.0005 & -0.0006 & 0.0025 & -0.0018 & 0.0022 & 0.0164 & -0.0047 & -0.0031 & 0.0025 & 0.5250 & 0.0186 & 0.55954 \\
\hline 8. & $\begin{array}{l}\text { No. seeds } \\
\text { per pod }\end{array}$ & -0.0020 & -0.0013 & -0.0004 & -0.0009 & 0.0004 & -0.0002 & 0.0042 & -0.0183 & -0.0010 & 0.0045 & 0.0934 & 0.0241 & 0.10258 \\
\hline 9. & $\begin{array}{c}\text { Seed } \\
\text { Index }\end{array}$ & 0.0016 & 0.0003 & 0.0019 & -0.0008 & -0.0001 & -0.0007 & -0.0074 & 0.0027 & 0.0069 & 0.0018 & 0.0755 & $\begin{array}{c}- \\
0.0094\end{array}$ & 0.07225 \\
\hline 10. & $\begin{array}{c}\text { Days to } \\
\text { 50\% Pod } \\
\text { Setting }\end{array}$ & -0.0016 & 0.0014 & 0.0002 & 0.0020 & 0.0002 & -0.0005 & -0.0031 & 0.0063 & -0.0009 & -0.0131 & 0.0555 & 0.0103 & 0.0567 \\
\hline 11. & $\begin{array}{c}\text { Biological } \\
\text { yield }\end{array}$ & 0.0079 & 0.0003 & 0.0020 & 0.0043 & -0.0032 & 0.0019 & 0.0094 & 0.0019 & 0.0006 & -0.0008 & 0.9211 & 0.0515 & 0.99658 \\
\hline 12. & $\begin{array}{l}\text { Harvest } \\
\text { Index }\end{array}$ & 0.0033 & 0.0004 & -0.0009 & 0.0023 & -0.0018 & 0.0007 & 0.0029 & -0.0042 & -0.0006 & -0.0013 & 0.4539 & 0.1044 & 0.55919 \\
\hline
\end{tabular}


Int.J.Curr.Microbiol.App.Sci (2019) 8(12): 1602-1608

Table.3 Estimation of path coefficient at phenotypic level for 13 yield component traits with grain yield per plant in chickpea during rabi, 2018

\begin{tabular}{|c|c|c|c|c|c|c|c|c|c|c|c|c|c|c|}
\hline No & Character & $\begin{array}{c}\text { Days to } \\
50 \% \\
\text { Flowering }\end{array}$ & $\begin{array}{l}\text { Days to } \\
\text { Maturity }\end{array}$ & $\begin{array}{c}\text { Plant } \\
\text { height }\end{array}$ & $\begin{array}{l}\text { Primary } \\
\text { branches }\end{array}$ & $\begin{array}{l}\text { Secondary } \\
\text { branches }\end{array}$ & $\begin{array}{l}\text { No. Pods } \\
\text { per plant }\end{array}$ & $\begin{array}{l}\text { No. seed } \\
\text { per plant }\end{array}$ & \begin{tabular}{c|} 
No. \\
seeds per \\
pod
\end{tabular} & $\begin{array}{c}\text { Seed } \\
\text { Index }\end{array}$ & $\begin{array}{l}\text { Days to } \\
50 \% \text { Pod } \\
\text { Setting }\end{array}$ & $\begin{array}{l}\text { Biological } \\
\text { yield }\end{array}$ & $\begin{array}{l}\text { Harvest } \\
\text { Index }\end{array}$ & $\begin{array}{l}\text { Seed } \\
\text { yield/ } \\
\text { plant }\end{array}$ \\
\hline 1. & $\begin{array}{c}\text { Days to } \\
50 \% \\
\text { Flowering }\end{array}$ & -0.0166 & 0.0002 & 0.0002 & -0.0007 & -0.0006 & -0.0039 & -0.0021 & -0.0009 & -0.0009 & 0.0002 & 0.4586 & -0.0167 & 0.41686 \\
\hline 2. & $\begin{array}{l}\text { Days to } \\
\text { Maturity }\end{array}$ & -0.0006 & 0.0040 & -0.0001 & 0.0002 & 0.0003 & -0.0016 & -0.0014 & 0.0021 & 0.0006 & 0.0001 & 0.0775 & 0.0095 & 0.0906 \\
\hline 3. & Plant height & 0.0020 & 0.0003 & -0.0013 & 0.0004 & 0.0006 & -0.0009 & -0.0009 & 0.0004 & 0.0023 & 0.0000 & 0.1934 & 0.0005 & 0.19663 \\
\hline 4. & $\begin{array}{l}\text { Primary } \\
\text { branches }\end{array}$ & 0.0028 & 0.0002 & -0.0001 & 0.0040 & 0.0008 & 0.0039 & 0.0026 & 0.0005 & -0.0008 & 0.0001 & 0.3716 & 0.0177 & 0.40315 \\
\hline 5. & $\begin{array}{l}\text { Secondary } \\
\text { branches }\end{array}$ & 0.0051 & 0.0006 & -0.0005 & 0.0018 & 0.0018 & 0.0035 & 0.0037 & 0.0005 & 0.0001 & 0.0000 & 0.5712 & 0.0337 & 0.62158 \\
\hline 6. & $\begin{array}{l}\text { No. Pods } \\
\text { per plant }\end{array}$ & 0.0068 & -0.0006 & 0.0001 & 0.0016 & 0.0007 & 0.0096 & 0.0079 & 0.0004 & -0.0024 & -0.0001 & 0.5729 & 0.0264 & 0.6232 \\
\hline 7. & $\begin{array}{c}\text { No. seed per } \\
\text { plant }\end{array}$ & 0.0032 & -0.0005 & 0.0001 & 0.0010 & 0.0006 & 0.0070 & 0.0107 & -0.0016 & -0.0043 & -0.0001 & 0.5129 & 0.0197 & 0.54872 \\
\hline 8. & $\begin{array}{l}\text { No. seeds } \\
\text { per pod }\end{array}$ & -0.0022 & -0.0012 & 0.0001 & -0.0003 & -0.0001 & -0.0005 & 0.0025 & -0.0069 & -0.0015 & -0.0001 & 0.0792 & 0.0177 & 0.0868 \\
\hline 9. & $\begin{array}{c}\text { Seed } \\
\text { Index }\end{array}$ & 0.0015 & 0.0002 & -0.0003 & -0.0003 & 0.0000 & -0.0023 & -0.0047 & 0.0010 & 0.0099 & 0.0000 & 0.0763 & -0.0104 & 0.07099 \\
\hline 10. & $\begin{array}{l}\text { Days to } \\
50 \% \text { Pod } \\
\text { Setting }\end{array}$ & -0.0051 & 0.0008 & 0.0001 & 0.0005 & -0.0001 & -0.0011 & -0.0013 & 0.0013 & -0.0008 & 0.0005 & 0.0209 & 0.0172 & 0.03276 \\
\hline 11. & $\begin{array}{l}\text { Biological } \\
\text { yield }\end{array}$ & 0.0082 & 0.0003 & -0.0003 & 0.0016 & 0.0011 & 0.0060 & 0.0060 & 0.0006 & 0.0008 & 0.0000 & 0.9257 & 0.0386 & 0.98856 \\
\hline 12. & $\begin{array}{c}\text { Harvest } \\
\text { Index }\end{array}$ & 0.0018 & 0.0003 & 0.0000 & 0.0005 & 0.0004 & 0.0017 & 0.0014 & -0.0008 & -0.0007 & 0.0001 & 0.2370 & 0.1507 & 0.39231 \\
\hline
\end{tabular}


The phenotypic correlation with Days to $50 \%$ flowering showed positive significant association with grain yield per plant $\left(0.417^{* *}\right)$, Days to maturity showed positive significant association with days to $50 \%$ pod setting $\left(0.200^{* *}\right)$, Plant height showed positive significant association with number of secondary branches $\left(0.346^{* *}\right)$, seed index $\left(0.226^{* *}\right)$, biological yield $\left(0.209^{* *}\right)$ and grain yield per plant $\left(0.197^{* *}\right)$, Number of number of panicles per hill showed positive significant association with number of secondary branches $\left(0.458^{* *}\right)$, number of pods per plant $(0.403 * *)$, number of seeds per plant $\left(0.238^{* *}\right)$, biological yield $\left(0.401^{* *}\right)$ and grain yield per plant $\left(0.403^{* *}\right)$, Number of secondary branches showed positive significant association with number of pods per plant $\left(0.362^{* *}\right)$, number of seeds per plant $\left(0.347^{* *}\right)$, biological yield $\left(0.617^{* *}\right)$, harvest index $\left(0.223^{* *}\right)$ and grain yield per plant $\left(0.622^{* *}\right)$, Number of pods per plant showed positive significant association with number of seeds per plant $(0.732 * *)$, biological yield $\left(0.619^{* *}\right)$, harvest index $\left(0.175^{* *}\right)$ and grain yield per plant $\left(0.623^{* *}\right)$, Number of seeds per plant showed positive significant association with number of seeds per pod $\left(0.237^{* *}\right)$, biological yield $\left(0.554^{* *}\right)$ and grain yield per plant $(0.549 * *)$, Biological yield showed positive significant association with harvest index $\left(0.256^{* *}\right)$ and grain yield per plant $\left(0.989^{* *}\right)$, Harvest index showed positive significant association with grain yield per plant $(0.392 * *)$. These traits seem to be major yield factors, hence selection of these characters will be effective for improvement on seed yield. These findings are in accordance with (Table 1).

Path coefficient analysis is the most widely used biometrical technique in plant breeding. The information obtained from this technique, also helps in making selection based on component characters of yield. It helps in understanding the cause of association between two variables. Traits viz., The path analysis at genotypic level indicated that days to $50 \%$ flowering (0.0134), days to maturity (0.0036), plant height (0.0051), number of primary branches (0.0098), number of pods per plant (0.0029), number of seeds per plant (0.0164), seed index (0.0069), biological yield $(0.921)$ and harvest index (0.1044) showed positive direct effect on grain yield per plant.

The path analysis at phenotypic level indicated that days to maturity (0.0040), number of primary branches (0.0040), number of secondary branches $(0.0018)$, number of pods per plant (0.0096), number of seeds per plant (0.0107), seed index (0.0099) days to 50\% pod setting (0.0005), biological index (0.9257) and harvest index (0.1507) showed positive direct effect on grain yield per plant (Table 2 and 3 ).

\section{References}

Jeena, A. S. and Arora, P. P. 2002. Path analysis in relation to selection in chickpea. Agricultural Science Digest, 22 (2): $132-133$.

Jeena, A. S. and Arora, P. P. and Ojha, O. P. 2005. Variability and correlation studies for yield and its components in chickpea. Legume Res., 28(2): 146 148.

Jivani, L.L. and Yadavendra, S.P. (1988). Genetic variability in chickpea. Indian Journal of Pulses Res., 1: 62-63.

Kuldeep, R., Pandey, S., Babbar, A. and Mishra, D.K. 2014. Genetic variability, character association and path coefficient analysis in chickpea grown under heat stress conditions. Electronic Journal of Plant Breeding. 5(4): 812819.

Kumar, A.,G., Suresh Babu and Roopa Lavanya, $\quad$ G. 2012. Characterassociation and path analysis in early segregating population in chickpea (Cicer arietinum L.). Legume 
Res., 35 (4): 337.

Kumari, N., Babu, S. and Lavanya, G.R. (2013). Genetic variability and character association in Chickpea germplasm (Cicer arietinium L.). Trends in Biosciences, 6(6):742-743

Mushtaq, M. A., Bajwa, M. M., and Saleem, M. 2013. Estimation of genetic variability and path analysis of grain yield and its components in chickpea. International Journal of Scientific and Engineering Research, Vol. 4 (1).

Nizama, J. R., S. R. Patel, A. I. Patel (2013). Genetic Variability and Heritability Among Quantitative Traits in Chickpea Under Tropical Region. Asian Resonance. VOL.-II, ISSUE-IV.
Sood, B.C. and Kumari, V. (2000). Genetic variability and characters associations in kabuli gram. Indian Journal of Pulses Res., 13(1): 48-49.

Yadav, P., Tripathi, D., Khan, K. K., Yadav, A. 2012. Character association and path coefficient analysis in chickpea (Cicer arietinum L.) under late sown conditions. Forage Res., 37 (4): pp. 258 -262 .

Yucel, D. O. and Anlarsal, A. E., Yucel, C. 2006. Genetic variability, correlation and path analysis of yield and yield contributing characters in chickpea (Cicer arietinum L.). Turk J.Agric For30: 183 - 188.

\section{How to cite this article:}

Bijjamwar Makarand, G. M. Lal and Karthik Manikanta Sai, I. 2019. Estimation of Correlation and Path Analysis for Quantitative traits in Chickpea (Cicer arientinum L.). Int.J.Curr.Microbiol.App.Sci. 8(12): 1602-1608. doi: https://doi.org/10.20546/ijcmas.2019.812.192 